\title{
Functional Validation of microRNA-126-3p as a Platelet Reactivity Regulator Using Human Haematopoietic Stem Cells
}

\author{
Alix Garcia ${ }^{1}$ Sylvie Dunoyer-Geindre ${ }^{1}$ Veronika Zapilko ${ }^{1} \quad$ Séverine Nolli ${ }^{1}$ Jean-Luc Reny ${ }^{1,2}$ \\ Pierre Fontana ${ }^{1,3}$ \\ ${ }^{1}$ Geneva Platelet Group, Faculty of Medicine, University of Geneva, \\ Geneva, Switzerland \\ 2 Division of General Internal Medicine, Geneva University Hospitals, \\ Geneva, Switzerland \\ ${ }^{3}$ Division of Angiology and Haemostasis, Geneva University \\ Address for correspondence Pierre Fontana, MD, PhD, Division of \\ Angiology and Haemostasis, University Hospitals of Geneva, Rue \\ Gabrielle-Perret-Gentil 4, CH-1205 Geneva, Switzerland \\ (e-mail: Pierre.Fontana@hcuge.ch).
}

Hospitals, Geneva, Switzerland

Thromb Haemost 2019;119:254-263.

\section{Abstract}

Keywords

- microRNA

- platelet

- platelet function tests

- flow-based assay

- PLXNB2

Background Platelets are an abundant source of micro-ribonucleic acids (miRNAs) that may play a role in the regulation of platelet function. Some miRNAs, such as miR126-3p, have been noted as potential biomarkers of platelet reactivity and the recurrence of cardiovascular events. However, the biological relevance of these associations remains uncertain, and the functional validation of candidate miRNAs on human-derived cells is lacking.

Objective This article functionally validates miR-126-3p as a regulator of platelet reactivity in platelet-like structures (PLS) derived from human haematopoietic stem cells.

Materials and Methods $\mathrm{CD} 34^{+}$-derived megakaryocytes were transfected with miR126-3p and differentiated in PLS. PLS reactivity was assessed using perfusion in a fibrinogen-coated flow chamber. miR-126-3p's selected gene targets were validated using quantitative polymerase chain reaction, protein quantification and a reporter gene assay.

Results CD34+-derived megakaryocytes transfected with miR-126-3p generated PLS exhibiting $156 \%$ more reactivity than the control. These functional data were in line with those obtained analysing CD62P expression. Moreover, miR-126-3p transfection was associated with the down-regulation of a disintegrin and metalloproteinase- 9 (ADAM9) messenger RNA (mRNA), a validated target of miR-126-3p, and of Plexin B2 (PLXNB2) mRNA and protein, an actin dynamics regulator. Silencing PLXNB2 led to similar functional results to miR-126-3p transfection. Finally, using a reporter gene assay, we validated PLXNB2 as a direct target of miR-126-3p.

Conclusion We functionally validated miR-126-3p as a regulator of platelet reactivity in PLS derived from human haematopoietic stem cells. Moreover, PLXNB2 was validated as a new gene target of miR-126-3p in human cells, suggesting that miR-126-3p mediates its effect on platelets, at least in part, through actin dynamics regulation.
\end{abstract}

received

June 7, 2018

accepted after revision

November 7, 2018
DOI https://doi.org/

$10.1055 / \mathrm{s}-0038-1676802$.

ISSN 0340-6245. (c) 2019 Georg Thieme Verlag KG Stuttgart · New York
License terms

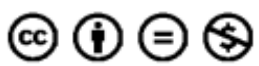




\section{Introduction}

Platelets play a crucial role, not only in haemostasis but also in inflammation, cell proliferation and immune system modulation. ${ }^{1-3}$ Identifying the determinants of platelet reactivity may thus have clinical implications on the prognoses for several diseases and may help to tailor drug treatments. The discovery that platelets are an abundant source of microribonucleic acids (miRNAs) ${ }^{4}$ and that miRNA expression profiles within platelets correlate with platelet reactivity ${ }^{5,6}$ raised the exciting possibility of finding novel disease biomarkers and therapeutic targets. ${ }^{7,8}$

The vast majority of studies designed to address the role of miRNAs in platelet reactivity regulation are association-type studies investigating correlations between miRNA levels and platelet reactivity scores or the recurrence of ischaemic events. A functional approach is needed to understand the mechanisms involved and to validate the role of miRNAs in platelet function. Several studies have described methods using animal models ${ }^{9,10}$ and immortalized cell lines, ${ }^{11,12}$ but the functional validation of candidate miRNAs in humanderived cells is lacking. However, studying the function of miRNAs in anucleated platelets that are refractory to transfection is a major limitation. ${ }^{13}$

Platelet-like structures (PLS) derived from megakaryocytes can be used as a model to identify the functional impact of miRNAs on platelet reactivity. Over the last few years, several research groups have focused on producing PLS with the aim of creating in vitro transfusable platelets. PLS produced from haematopoietic stem cells are functionally close to human platelets. ${ }^{14-18}$ Indeed, the secretion of PLS after agonist stimulation, as well as their function in relation to fibrinogen receptors, is comparable to the results observed with human platelets. ${ }^{19,20}$

Microfluidic flow chambers are currently used to study thrombotic processes by monitoring platelet incorporation into the clot, to study cell adhesion in dynamic conditions or to test the function of blood bank platelets in whole or reconstituted blood. ${ }^{21,22}$ This process is recognized as a powerful, promising method to investigate platelet function.

This study describes the use of a flow-based assay, using PLS derived from human haematopoietic stem cells, to assess the functional impact of candidate miRNAs for regulating platelet reactivity. As a functional proof-of-concept experiment, we used miR-126-3p, one of the most promising platelet-derived miRNAs associated with platelet reactivity ${ }^{11}$ and a potential biomarker of recurring cardiovascular events in humans. ${ }^{23}$

\section{Materials and Methods}

\section{Cell Culture and Differentiation}

Human $\mathrm{CD}_{3} 4^{+}$cells were isolated from the buffy coats of healthy adult human donors provided by the Geneva University Hospitals' blood bank using a CD34 MicroBead Kit (Miltenyi Biotec, Bergisch Gladbach, Germany). CD34 ${ }^{+}$cell quantification was evaluated using an anti-CD34 PE antibody (Miltenyi Biotec) followed by flow cytometry analysis. More than $95 \%$ of the cells were positive for CD34. CD34 ${ }^{+}$cells were cultured for 7 days in
StemSpan Serum-Free Expansion Medium (Stemcell Technologies, Vancouver, Canada) supplemented with $20 \mathrm{ng} / \mathrm{mL}$ human low-density lipoprotein (LDL, Stemcell Technologies), StemSpan Megakaryocyte Expansion Supplement (CC220, Stemcell Technologies), penicillin-streptomycin-glutamine (PSG, Gibco, Thermofisher, Waltham, Massachusetts, United States) and $1 \mu$ M StemRegenin 1 (SR1, Cellagen Technology, San Diego, California, United States), as described elsewhere. ${ }^{15}$ Next, the cells were washed, seeded and cultured in the presence of $0.5 \mu \mathrm{g} / \mathrm{mL}$ thrombopoietin (TPO, Stemcell Technologies), LDL, PSG and SR1 for an additional 8 days (-Supplementary Fig. S1A, available in the online version).

All the experiments described below were repeated with different, independent batches of cells from different blood bank donors.

\section{Flow Cytometry}

Megakaryocytes and PLS were analysed using flow cytometry with selected antibodies against specific markers of cell differentiation (CD34, CD41, CD42b and CD42d).

Anti-CD34 PE (Miltenyi Biotec), anti-CD41 fluorescein isothiocyanate (BioLegend, San Diego, California, United States) and anti-CD42b PE-vio770 (Miltenyi Biotec) antibodies were added directly to the cell suspension for 20 minutes, at room temperature, in the dark, and were then washed and re-suspended in phosphate-buffered saline.

Anti-CD42d mouse primary antibody (Santa Cruz, Dallas, Texas, United States) was added to the cells for 20 minutes. The cells were washed and then incubated with the secondary PE goat anti-mouse antibody (BioLegend) for 20 minutes.

Flow cytometry was performed using Attune (ThermoFisher, Ecublens, Switzerland) and Accuri C6 (BD Biosciences, Allschwil, Switzerland) cytometers. Positive cells were defined using appropriate isotypic control. The data acquired were analysed using the FlowJo software (TreeStar, Ashland, Oregon, United States).

\section{Isolation and Quantification of Megakaryocytes and PLS}

Megakaryocytes were isolated at day (D) 13 using a 10minute centrifugation step at $400 \times \mathrm{g}$. We used a Tali ImageBased Cytometer (ThermoFisher) to quantify megakaryocytes defined as elements $>6 \mu \mathrm{m}$.

At D15, PLS and megakaryocytes were quantified using the Tali Image-Based Cytometer; PLS were defined as elements $\geq 4$ and $\leq 6 \mu \mathrm{m}$. The number of PLS was then normalized to the number of megakaryocytes to evaluate production.

\section{Activation of PLS}

Non-PLS cells were discarded at D15 using a 5-minute centrifugation step at $100 \times g$ after addition of $25 \mu \mathrm{M}$ prostaglandin $\mathrm{I}_{2}$ (Cayman Chemical Company, Ann Arbor, Michigan, United States) and $0.02 \mathrm{U} / \mathrm{mL}$ apyrase (Sigma, St. Louis, Missouri, United States). The supernatant was centrifuged at $1,000 \times \mathrm{g}$ for 10 minutes and PLS were re-suspended in Tyrode's buffer (5.5 mM glucose, $137 \mathrm{mM} \mathrm{NaCl}$, $2 \mathrm{mM} \mathrm{KCl}, 12 \mathrm{mM} \mathrm{NaHCO}, 0.3 \mathrm{NaH}_{2} \mathrm{PO}_{4}, 5 \mathrm{mM}$ HEPES, $1 \mathrm{mM} \mathrm{MgCl}_{2}$ and $2 \mathrm{mM} \mathrm{CaCl}_{2}$; $\mathrm{pH}$ was adjusted to 7.3). After 
1 hour at $37^{\circ} \mathrm{C}$, PLS were stimulated with $1 \mathrm{U} / \mathrm{mL}$ thrombin (Sigma) and stirred for 10 minutes. PLS were labelled with CD62P PE antibody (BD Biosciences) and CD41 APC (BioLegend). PLS labelled with an isotypic antibody (PE, BD Biosciences, and APC, BioLegend) were used to define positive cells. Flow cytometry was performed using an Accuri C6 flow cytometer (BD Biosciences), and the data were analysed using the FlowJo software (TreeStar).

\section{miR-126-3p Expression Profile during Differentiation and after Transfection}

On selected culture days, cells were recovered in QIAzol lysis reagent (Qiagen, Hilden, Germany). Isolation of RNAs using the miRNeasy Mini Kit (Qiagen) was followed by a reverse transcription procedure using the TaqMan Advanced miRNA cDNA Synthesis Kit (Applied Biosystems, Foster City, California, United States). miRNAs were detected in triplicate on a 7900HT Sequence Detection System using TaqMan Advanced miRNA assays (Applied Biosystems) with pre-designed miRNAs probes (Applied Biosystems). miRNAs were quantified by normalizing miRNA reverse transcriptase-polymerase chain reaction (RT-PCR) data using a panel of stably expressed miRNAs. Using the geNorm algorithm, three of the six miRNAs constituting the platelet normalization panel given by Kok et $\mathrm{al}^{24}$-miR28, miR151 and miR29c-were identified as the best combination of normalizers in megakaryocytes and these were assessed in each sample. ${ }^{25}$

The transfection procedure's impact on miR-126-3p and miR-223-3p (a control platelet-derived miRNA) expression was quantified at D15 in PLS only.

\section{Cell Transfection}

Megakaryocytes isolated at D13 were seeded in an antibioticfree medium at $1 \times 10^{6}$ cells per well for 2 hours in 6 -well plates. They were then transfected at $200 \mathrm{nM}$ using an hsamiR-126-3p mimic, or scramble miRNA (ThermoFisher), which does not target any specific messenger RNA (mRNA), using Lipofectamine 2000 Transfection Reagent (Invitrogen, Carlsbad, California, United States). Plexin B2 (PLXNB2) small interfering RNA (siRNA) (SI00687603, Qiagen) or scramble siRNA were transfected at $50 \mathrm{nM}$ using RNAiMAX Transfection Reagent (Invitrogen). Five hours after transfection, the medium was replaced and the cells were resuspended in StemSpan supplemented with $0.5 \mu \mathrm{g} / \mathrm{mL}$ TPO, $20 \mathrm{ng} / \mathrm{mL}$ LDL and $1 \mu \mathrm{M}$ SR1. Results were compared to those of the mock condition (cells with transfection reagent only).

Transfection procedure efficiency was assessed at D13, 5 hours after megakaryocyte transfection, using siRNA AllStars negative Alexa Fluor 488 (Qiagen). The percentage of transfected cells was measured using Accuri C6 flow cytometer.

\section{Flow Chamber Assay}

Flow chambers (Vena8fluoro + , Cellix, Ireland) were coated overnight with $200 \mu \mathrm{g} / \mathrm{mL}$ fibrinogen (Sigma) at $4^{\circ} \mathrm{C}$ and washed with StemSpan Serum-Free Expansion Medium (Stemcell Technologies) for 1 minute at $80 \mu \mathrm{L} / \mathrm{min}$. At D15, the cell suspension ( $44 \pm 5.2 \%$ PLS, the rest being $\mathrm{CD}^{2} 4^{+}$derived megakaryocytes) was adjusted to $5 \times 10^{6}$ cells $/ \mathrm{mL}$.
The cell suspension was perfused for 5 minutes at $2 \mu \mathrm{L} / \mathrm{min}$ (shear rate of $50 \mathrm{~s}^{-1}$ ) and rinsed for 8 minutes at $3 \mu \mathrm{L} / \mathrm{min}$ using the StemSpan medium. Pictures of each channel were taken at five different points along them, $4 \mathrm{~mm}$ apart, with picture \#1 closest to the injection site, using a Si-3000 camera and XLi Cap V18 driver software (Ceti, Medline Scientific, Chalgrove, England).

Flow chamber images were analysed using a custommade software framework written in MATLAB (The MathWorks, Inc. Natick, Massachusetts, United States). Briefly, RGB images were first converted into greyscale. The flow area was then automatically detected within the field of view of the images; this enabled calibration of the image resolution in accordance with the chamber's $400 \mu \mathrm{m}$ width. The content of the flow area was subsequently segmented using Otsu's method, ${ }^{26}$ preceded by a background subtraction through a combination of low-pass filters. Separation of the multiobject content was done using watershed transformations ${ }^{27}$ constrained by the regional extrema within the aggregate. This approach is also effective in cases involving single cells. Finally, each resulting individual object was classified by size as either a megakaryocyte cell $(>6 \mu \mathrm{m})$, a PLS ( $\geq 4$ and $\leq 6$ $\mu \mathrm{m})$ or debris $(<4 \mu \mathrm{m})$. The number of PLS adhered to the immobilized fibrinogen was quantified and normalized to the mock condition.

\section{Gene Expression Levels of Selected miR-126-3p Gene Targets}

Forty-eight hours after transfection (D15), mRNA was extracted from the PLS using the trizol procedure. After RNA isolation, total RNA was reverse-transcribed using Improm-II reverse transcriptase (Promega, Madison, Wisconsin, United States) according to the manufacturer's instructions. A quantitative real-time RT-PCR (qPCR) was carried out using the $\Delta \Delta C_{t}$ method to evaluate the levels of a disintegrin and a metalloproteinase-9 (ADAM9) and PLXNB2 mRNAs. The level of HB2M was used as a housekeeping gene to normalize the qPCR results ( - Table $\mathbf{1}$ ).

\section{Western Blot}

A Western blot analysis was performed 48 hours after transfection (D15) to quantify the PLXNB2 protein. Because the amount of protein in the PLS was too low, we performed

Table 1 Oligonucleotide sequences used in this study

\begin{tabular}{|l|l|l|}
\hline Gene & Direction & Sequence \\
\hline HB2M & Forward & TGCTCGCGCTACTCTCTCTTT \\
\hline HB2M & Reverse & TCTGCTGGATGACGTGAGTAAAC \\
\hline ADAM9 & Forward & CCCCCAAATTGTGAGACTAAAG \\
\hline ADAM9 & Reverse & TCCGTCCCTCAATGCAGTAT \\
\hline PLXNB2 & Forward & GAAGACACCATCCACATC \\
\hline PLXNB2 & Reverse & $\begin{array}{l}\text { ACTGAACCTGACCGTACAATGC } \\
\text { ACGTCAAAGATGAAG }\end{array}$ \\
\hline PmirGLO & Forward & GAGCTCTAGCGGCCGCCCTAC \\
\hline PmirGLO & Reverse & CCTGCAGGCAGAGTGAAAAAGAC \\
\hline
\end{tabular}


this quantification in PLS and megakaryocytes. Proteins were labelled with an anti-PLXNB2 (AF5329, R\&D, Minneapolis, Minnesota, United States) and an anti- $\beta$-actin antibody (Sigma). Horseradish peroxidase-conjugated secondary antibody (BioRad, Hercules, California, United States, and Abcam, Cambridge, England) was used for detecting chemiluminescence in a myECL imager (ThermoFisher). Band intensities were quantified using the ImageJ software (http//imagej.nih. gov/ij), and the PLXNB2 protein level was normalized to $\beta$ actin.

\section{Reporter Gene Assay}

The 3' untranslated region (UTR) of PLXNB2 mRNA, containing the miR-126-3p binding sequence wild-type (wt) or a mutant (mut) form (Invitrogen), was inserted into pmiRGLO Dual-Luciferase miRNA Target Expression Vector (Promega). The final sequences were validated using deoxyribonucleic acid sequencing (-Table 1 ). The empty pmirGLO plasmid containing no UTR sequence served as a negative control. HT1080 cells were plated in 96-well plates at 8,000 cells per well and co-transfected with pmirGLO-3'-UTR-wt, or pmirGLO-3'-UTR-mut and miRNA (50 nM scramble, $50 \mathrm{nM}$ miR-126-3p mimic or $50 \mathrm{nM}$ miR-126-3p inhibitor, which shares a complementary sequence with the miRNA mimic; ThermoFisher), using the Lipofectamine LTX and Plus $^{\mathrm{M}}$ reagent (Life Technologies). Each transfection was done in quadruplicate. After 48 hours, luciferase assays were carried out using the Dual-Luciferase Reporter Assay System (Promega). Individual luciferase activity was normalized to the corresponding Renilla luciferase activity constitutively expressed in the pmirGLO vector. Luminescence was measured using a Perkin Elmer Victor3 luminescence detection instrument (Perkin Elmer, Waltham, Massachusetts, United States).

\section{Statistics}

Data are expressed as means and standard error of the mean. The paired Student's $t$-test or the one-way analysis of variance followed by Dunnett's multiple comparison test was performed, when appropriate. Data were analysed using GraphPad Prism 7 software (GraphPad Software Inc., USA). $p$-Values of $<0.05$ were considered statistically significant.

\section{Results}

\section{Haematopoietic Stem Cell Differentiation}

$\mathrm{CD}_{4} 4^{+}$cells were cultured for 7 days before induction of differentiation via the addition of TPO. At D7, $69 \pm 12.3 \%$ of the cells were positive for $\mathrm{CD} 34$, and $26 \pm 4.1,16 \pm 2.8$ and $17 \pm 4.1 \%$ were positive for the CD41, CD42b and CD42d markers of differentiation, respectively. As expected, after induction of differentiation, the percentage of $\mathrm{CD} 34^{+}$cells had decreased at D15 to $42 \pm 6.4 \%$, whereas cells positive for CD41, CD42b and CD42d had increased to $86 \pm 0.7,79 \pm 2.0$ and $74 \pm 6.2 \%$, respectively (-Supplementary Fig. S1B, available in the online version).

Finally, we investigated the time-course pattern of miR126-3p levels during human haematopoietic stem cell differ-

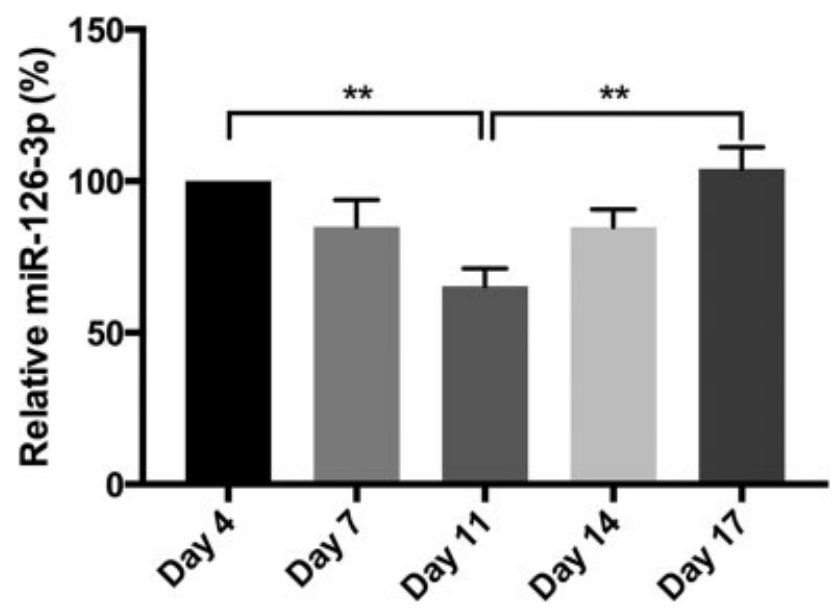

Fig. 1 Expression level of miR-126-3p during differentiation. The expression level of miR-126-3p is represented relative to the level in CD34 ${ }^{+}$cells after 4 days of culture $(n=4) .{ }^{* *} p<0.01$.

entiation. As shown in -Fig. 1, the level of miR-126-3p decreased between D4 and D11, and then increased back to its initial level at D17.

\section{Transfection Procedure Efficiency}

The transfection procedure was performed at D13. Tested 5 hours after the procedure, using siRNA labelled with Alexa Fluor 488, transfection efficiency was of $47 \pm 3.2 \%(n=3$, - Supplementary Fig. S2A, available in the online version). In the PLS, miR-126-3p expression had increased by a factor of $2.4 \pm 0.28$ more than the mock condition (-Supplementary Fig. S2B) 48 hours after the transfection procedure, without affecting either the differentiation process ( - Supplementary Fig. S2C, available in the online version) or the production of PLS ( - Supplementary Fig. S2D, available in the online version), which was of $4.1 \pm 4.210^{5} \mathrm{PLS} / \mathrm{mL}$ from $1 \times 10^{6}$ megakaryocytes/mL at D13 in the mock condition ( - Supplementary Fig. S2D, available in the online version). Furthermore, transfection of miR-126-3p did not affect miR-223-3p level, one of the main platelet-associated miRNAs (- Supplementary Fig. S2E, available in the online version).

\section{Functional Impact of Over-Expression of miR-126-3p on Platelet-Like Structures}

At D15, corresponding to the end of megakaryocyte differentiation and maturation, the functional impact of miR-126$3 p$ on the reactivity of PLS was assessed 48 hours after miR126-3p transfection. A cell suspension containing mature megakaryocytes and PLS was perfused for 5 minutes at a shear rate of $50 \mathrm{~s}^{-1}$ in a microfluidic device coated with fibrinogen. The flow chamber assay showed that the number of PLS immobilized in the channel increased by $156 \pm 14.9 \%$ after miR-126-3p transfection compared to the mock condition (-Fig. 2A and B). Moreover, in the PLS over-expressing miR-126-3p, we observed a 30\% greater increase in CD62P expression after thrombin activation ( $\mathbf{- F i g . ~ 2 C ) ~ i n ~ c o m p a r - ~}$ ison to the mean fluorescence after activation seen in the mock condition. 

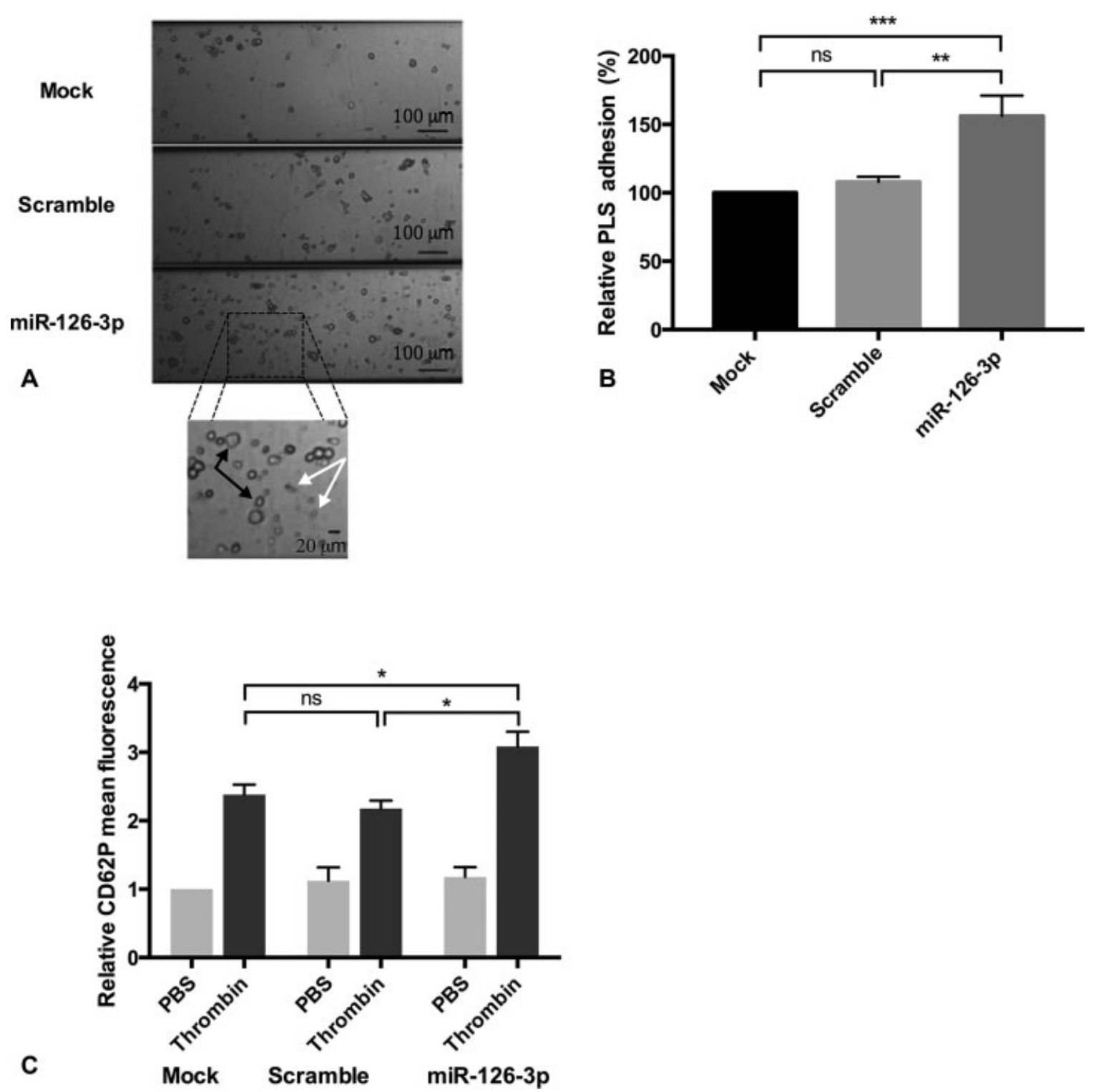

Fig. 2 Effect of miR-126-3p overexpression on megakaryocytes and platelet-like structure (PLS). (A) Inverted microscopy photographs of PLS and CD34 ${ }^{+}$-derived megakaryocytes in a fibrinogen-coated channel. Black arrows: megakaryocytes; white arrows: PLS. (B) miR-126-3p transfection increased the adhesion of PLS in a fibrinogen-coated channel $(n=12)$. Results are expressed relative to the mock condition. ${ }^{* * *} p<0.001,{ }^{* *} p<0.01$. (C) CD62P relative mean of fluorescence of PLS before and after activation using thrombin $1 \mathrm{U} / \mathrm{mL}$, compared to mock condition $(\mathrm{n}=3)$. ${ }^{*} \mathrm{p}<0.05$.

\section{miR-126-3p Targets ADAM9 and PLXNB2 Genes in Platelet-Like Structures Derived from Human Haematopoietic Stem Cells}

ADAM9 is a known miR-126-3p target, as previously shown in mouse and human immortalized cell lines. ${ }^{11,28}$ In the PLS derived from human $\mathrm{CD} 34^{+}$cells, we also observed that miR126-3p transfection was associated with a $30 \pm 3.7 \%$ downregulation of ADAM9 mRNA levels compared to the mock condition (-Fig. 3A).

To screen for further putative miR-126-3p gene targets in human cells, we examined in silico databases and identified PLXNB2 as the best candidate, with its Context ++ score of 0.58 using TargetScan (http://www.targetscan.org) and an miRSVR score of -0.45 using miRANDA (http://www.microrna.org). In PLS, miR-126-3p over-expression was associated with a $29 \pm 2.6 \%$ down-regulation of the PLXNB2 mRNA level (-Fig. 3B) and a $30 \pm 6.1 \%$ down-regulation of the PLXNB2 protein level ( - Fig. $3 \mathbf{C}$ and $\mathbf{D}$ ).

Finally, silencing PLXNB2 in megakaryocytes induced a functional phenotype similar to that observed after transfection with miR-126-3p, and this was associated with a $148 \pm 11.8 \%$ increase in the number of PLS immobilized in the flow chamber in comparison to the mock condition (-Fig. 4).

Validation of PLXNB2 as a Direct Target of miR-126-3p To investigate the causal relationship between miR-126-3p and PLXNB2 down-regulation, a reporter gene assay was performed using the 3'UTR sequence of PLXNB2 wt or mut cloned into dualGLO luciferase reporter vector (-Fig. 5A). 

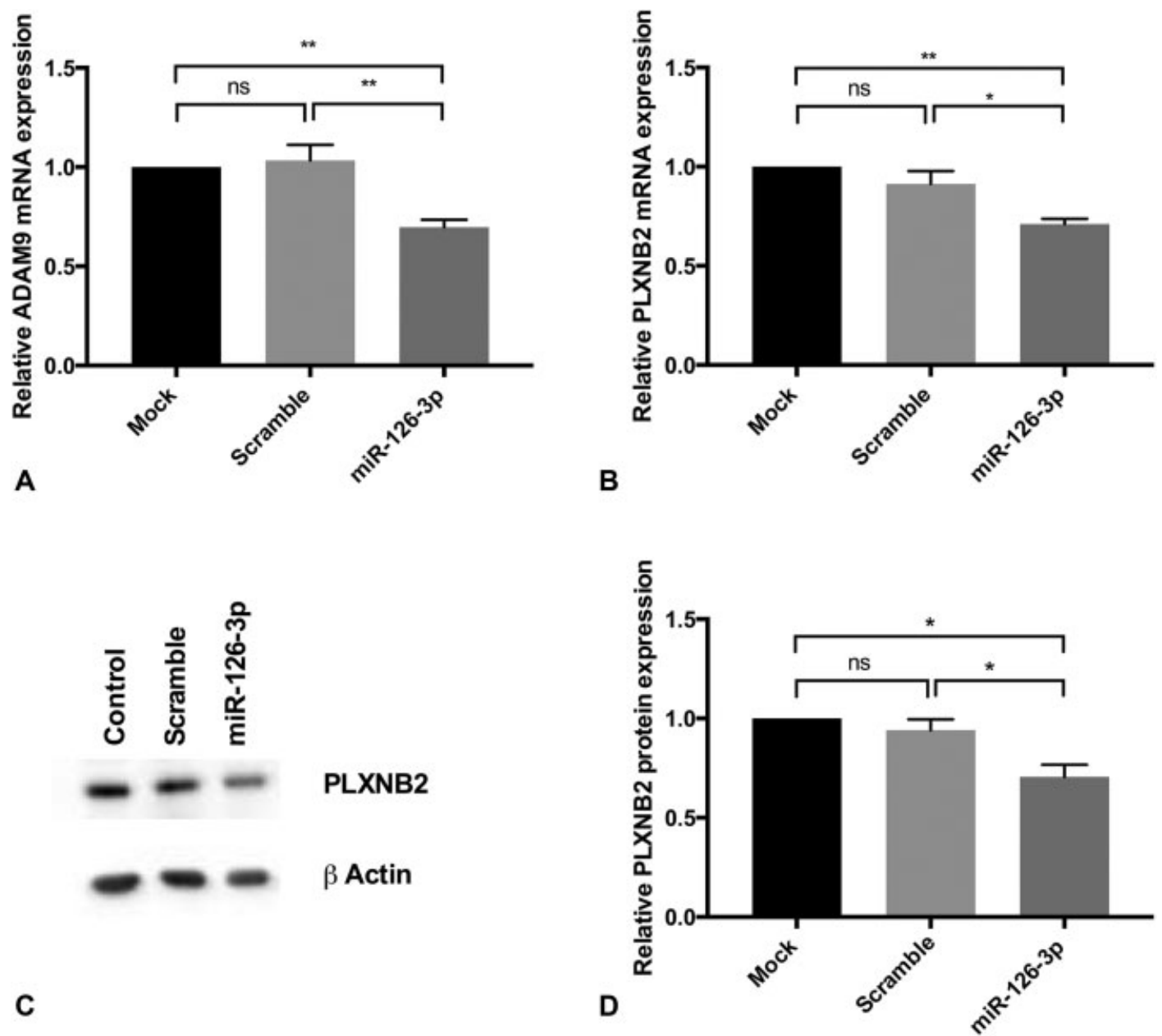

Fig. 3 Impact of miR-126-3p transfection on selected gene targets. Cell suspension was transfected at day (D) 13 using scramble microribonucleic acid (miRNA) or the miR-126-3p mimic, and messenger RNA (mRNA) was extracted at D15. (A) Relative expression levels of a disintegrin and metalloproteinase-9 (ADAM9) mRNA in platelet-like structure (PLS) in different conditions $(n=3) .{ }^{* *} p<0.01$. (B) Relative expression levels of Plexin B2 (PLXNB2) mRNA in PLS in different conditions $(n=3)$. ${ }^{*} p<0.05,{ }^{* *} p<0.01$. (C) Western blot of PLXNB2 protein after scramble or miR-126-3p transfection in megakaryocytes and PLS. The picture is representative of three independent experiments. (D) Quantification of PLXNB2 protein relative to the mock condition $(n=3) .{ }^{*} p<0.05$.

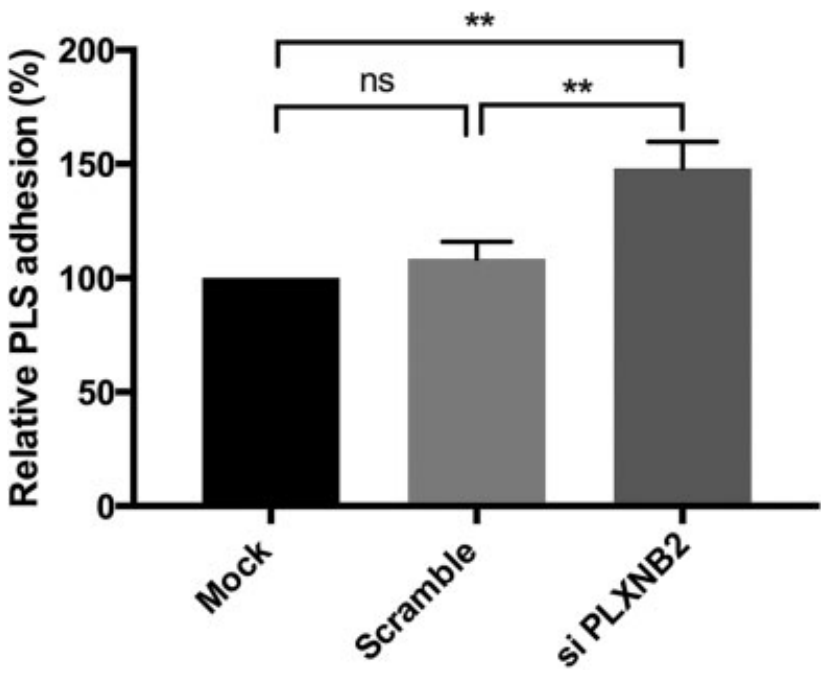

Fig. 4 Plexin B2 (PLXNB2) silencing modulates platelet-like structure (PLS) reactivity. Silencing PLXNB2 increased the adhesion of PLS on the fibrinogen-coated channel. Results are expressed relative to the mock condition $(n=6)$. ${ }^{* *} p<0.01$.
Co-transfection of HT1080 cells with pmirGLO-3'UTR PLXNB2 wt and miR-126-3p resulted in a $42 \pm 2.4 \%$ reduction in luciferase activity compared with cells transfected in the absence of miR-126-3p. Moreover, the addition of miR-126-3p inhibitor reversed the effect of the miR-126-3p mimic (- Fig. 5B). No significant difference in luciferase activity was observed upon transfection with scramble miRNA. Co-transfection of miR-126-3p and the pmirGLO mut did not lead to a significant change in luciferase activity ( - Fig. 5C).

\section{Discussion}

In this study, we demonstrated the feasibility of functionally validating a candidate miRNA of the regulation of platelet reactivity by using human-derived PLS and a flow chamber assay. Moreover, using this model, we validated PLXNB2 as a target gene of miR-126-3p and as a potential regulator of platelet reactivity.

PLS were derived from human haematopoietic stem cells following a protocol described elsewhere that allows the 


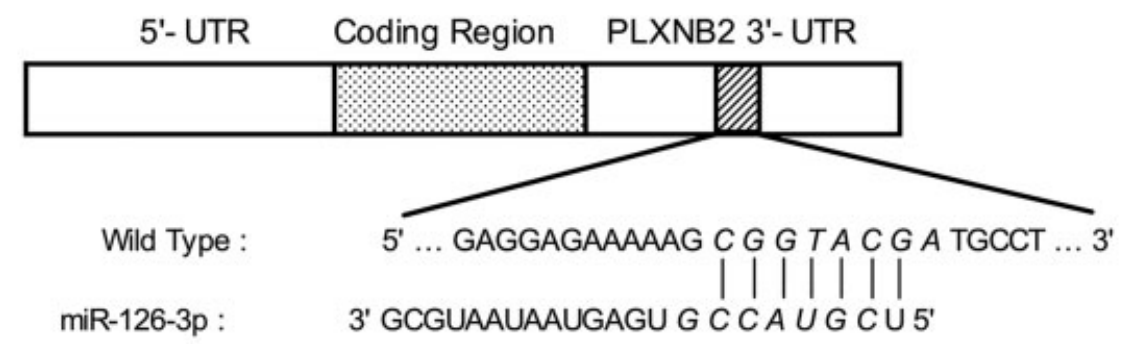

Mutant : $\quad 5^{\prime} \ldots$ GAGGAGAAAAAG G C TA T G C A TGCCT ... 3'

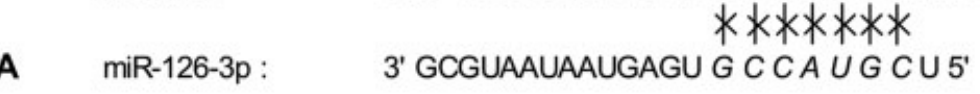

B

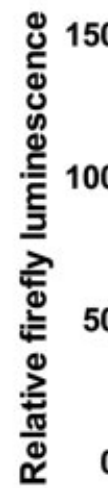

PLXNB2 UTR wt Scramble miR-126-3p mimic miR-126-3p inhibitor
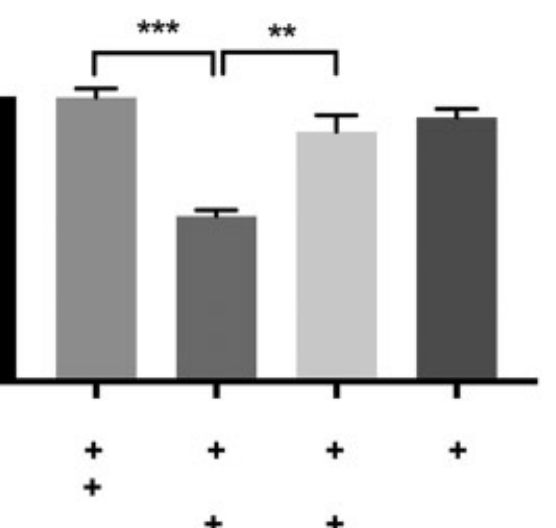

$+$ $+$

Fig. 5 3' Untranslated region (UTR) sequence of Plexin B2 (PLXNB2) is directly targeted by miR-126-3p. (A) The 3'UTR sequence of PLXNB2 wildtype (wt) and mutant (mut) were designed and cloned into PmirGLO vector. (B) The PmirGLO-3'UTR PLXNB2 wild-type was co-transfected with the miR-126-3p mimic and/or inhibitor in HT1080 cells. Luciferase activity was normalized on Renilla luminescence $(n=4)$. ${ }^{* *} p<0.01$,

${ }^{* * *} p<0.001$. (C) The PmirGLO-3'UTR PLXNB2 mutant was co-transfected with the miR-126-3p mimic and/or inhibitor in HT1080 cells $(n=4)$.

production of elements ultra-structurally and functionally similar to circulating platelets. ${ }^{15}$ At D15, $86 \%$ of cells were positive for $\mathrm{CD} 41$, and the proportions of $\mathrm{CD} 42 \mathrm{~b}$ and $\mathrm{Cd} 42 \mathrm{~d}$ positive cells were in line with those expected during the differentiation process. ${ }^{15}$ Moreover, the functional characterization of the PLS generated in this study using flow cytometry showed a 2.4-fold increase in CD62P expression after stimulation, in line with the twofold increase in glycoprotein (GP) IIb/IIIa activation ${ }^{15}$ and the 1.8 -fold increase in CD62P expression ${ }^{29}$ described in other studies. Finally, recent data have demonstrated significant correlations between the miRNA profile of primary human bone marrow megakaryocytes and cultured megakaryocytes derived from umbilical cord blood progenitors at D13. ${ }^{30}$ This further supports our approach based on in vitro haematopoietic stem cell culture and differentiation.

We quantified the miR-126-3p level during the differentiation procedure and observed an initial decrease from D4 to D11 followed by an increase up to D17. This U-shaped curve is in line with independent data on in vitro-differentiated megakaryocytes derived from $\mathrm{CD} 34^{+}$haematopoietic progenitors. This suggests that miR-126-3p may play a role in the regulation of the megakaryocyte differentiation process by unblocking
miR-126-3p's target genes during the proliferation stage (D7) and the early stages of differentiation (D11). ${ }^{31}$ The initial decrease in miR-126-3p was also evidenced during the first steps of mouse megakaryocyte differentiation. ${ }^{32}$ We thus speculate that the relatively low level of miR-126-3p could be associated with an up-regulation of the proteins involved in megakaryocyte differentiation.

It is of note that the transfection procedure with miR-126$3 p$ at D13 did not seem to alter the last stage of differentiation (D13-D15) since it had no impact on PLS production and the expression of differentiation markers.

Unstimulated PLS were perfused in a flow chamber assay, at a relatively low shear rate, to evaluate PLS adhesion on immobilized fibrinogen. Indeed, at a low shear rate, platelet immobilization is primarily mediated by fibrinogen through the GPIIb/IIIa receptor, the main receptor involved in the platelet aggregation process, ${ }^{33,34}$ whereas immobilization at higher shear rates relies more on a von Willebrand factordependent adhesion process. ${ }^{34}$ Using a higher shear rate (up to $1,000 \mathrm{~s}^{-1}$ ) in our assay was indeed associated with no PLS adhesion on the fibrinogen-coated flow chamber. It is of note that, instead of PLS only, we perfused a cell suspension that included both megakaryocytes and PLS through the flow 
chamber assay: this was to favour the margination process of PLS that is of the utmost importance in flow assays. ${ }^{35}$ In addition to the flow chamber assay results, miR-126-3p transfection was associated with a 30\% increase in CD62P expression in PLS after thrombin stimulation, compared to the mock condition. These data further support the existence of miR-126-3p-mediated platelet reactivity modulation and are in line with data showing a correlation between miR126-3p levels and soluble P-selectin in plasma. ${ }^{11}$

As expected, we observed that miR-126-3p transfection was associated with a $30 \%$ down-regulation in the level of ADAM9 mRNA compared to the mock control. This result was in line with data using locked nucleic acid and a miRNA mimic in a human megakaryoblastic cell line. ${ }^{11}$ Since immortalized cell lines can have different regulation mechanisms than cells derived from human progenitors, our result provides a valuable confirmation that ADAM9 is indeed a target gene for miR-126-3p in PLS derived from human haematopoietic stem cells.

In order to further investigate the mechanism associated with the increased reactivity of PLS mediated by miR-126-3p over-expression, we selected the most promising in silicopredicted gene target of miR-126-3p, which was PLXNB2. Interestingly, this gene has also been identified as differentially expressed in cardiovascular patients with an extreme platelet reactivity phenotype. ${ }^{6}$ We thus observed that miR-126-3p transfection induced a $29 \%$ down-regulation in PLXNB2 mRNA in PLS, and a 30\% down-regulation at the protein level in megakaryocytes and PLS. Moreover, the reporter gene assay validated that miR-126-3p directly regulates PLXNB2 gene expression, in line with recent data on ovarian cancer. ${ }^{36}$ Finally, transfection with miR-126-3p or the silencing of PLXNB2 using siRNA resulted in a similar functional phenotype. This suggests that miR-126-3p mediates its effect, at least in part, through the down-regulation of PLXNB2. The relative contributions of ADAM9, PLXNB2 or other gene targets to this hyper-reactivity phenotype are unknown.

Plexins-a family of transmembrane proteins-act as receptors for semaphorins, which control RhoGTPase signalling. There are several clues as to the possible roles of PLXNB2 and semaphorins in the regulation of actin dynamics. Semaphorin 4D, a PLXNB2 ligand, was recently shown to support communication between platelets in the early stages of thrombus formation in mice. ${ }^{37-39}$ In human glioblastoma cells, semaphorin $4 \mathrm{C}$ was shown to stimulate PLXNB2-inducing cellular actin dynamics regulation. ${ }^{40}$ In mouse macrophages, PLXNB2 negatively regulates the Rac1 and CDC42 Rho family proteins. ${ }^{38,41}$ Finally, by modulating Rho/Rac activity, PLXNB2 enables the modification of actin dynamics and, due to their effect on Ras/PI3K, they can modulate cell adhesion and migration. ${ }^{38}$ These observations support the hypothesis that the PLXNB2 gene might regulate platelet actin dynamics, and therefore platelet function, through the regulation of Rho family protein.

This work represents a significant step forward since, until now, the functional validation of miRNA in platelet physiology has been scarce. A first attempt was made in an animal model via the systemic administration of an antag-
omiR sequence against miR-126-3p in mice, followed by ex vivo platelet aggregation. ${ }^{11}$ However, predictive mRNA/ miRNA pairs are species-dependent, and mouse-model prediction does not always reflect human gene expression and regulation. ${ }^{42}$

It is worth noting that this study's main limitation is that its flow assay may not be suitable for the functional validation of all the candidate miRNAs regulating platelet reactivity: it will be dependent on the mechanism involved in the modulation of platelet reactivity.

In conclusion, we have described a method for the functional validation, using human-derived cells, of a candidate miRNA in the regulation of platelet reactivity. This model also enables the investigation of potential gene targets to improve our understanding of the regulation of platelet reactivity in humans. Using this method-and for the first time-we validated the impact of miR-126-3p and one of its targets, the PLXNB2 gene, as regulators of platelet function in human-derived cells.

\section{What is known about this topic?}

- MicroRNAs have been noted as potential biomarkers of the recurrence of cardiovascular events.

- miR-126-3p is a putative regulator of platelet reactivity.

- Functional validation of microRNAs in human-derived cells is lacking.

\section{What does this paper add?}

- We used a flow-based assay with platelet-like structures derived from human haematopoietic stem cells.

- We functionally validated miR-126-3p as a regulator of platelet reactivity using human cells.

- We validated PLXNB2 as a target gene of miR-126-3p, regulating the reactivity of platelet-like structures derived from human haematopoietic stem cells.

Authors' Contributions

A. Garcia, S. Dunoyer-Geindre, J.-L. Reny and P. Fontana designed the study and analysed the data. A. Garcia, S. Dunoyer-Geindre, S. Nolli and V. Zapilko performed the experiments and analysed the data. A. Garcia, S. DunoyerGeindre and P. Fontana wrote the first draft of the manuscript, and all authors revised the intellectual content and approved the final version.

\section{Funding}

This work was supported by the Swiss National Science Foundation (grant 310030_162567), the Novartis Foundation (grant 15B097) and the Swiss Heart Foundation.

\section{Conflict of Interest}

P. Fontana reports non-financial support from Novo Nordisk and Bayer, outside the scope of the submitted work. J.-L. Reny reports non-financial support from Bayer and 
grants from Daichii-Sankyo, outside the scope of the submitted work. The other authors have no conflicts of interest to report.

\section{Acknowledgements}

The authors wish to thank Catherine Strassel and Christian Gachet for helpful discussions and sharing protocols regarding the generation of $\mathrm{CD} 34^{+}$-derived megakaryocytes, as well as Nicolas Liaudet (Bioimaging core facility, University of Geneva) for the development of software dedicated to the quantification of the elements in the flow chamber and Mylène Docquier (iGE3 Genomics platform, University of Geneva) for the development of method allowing miRNAs quantification. We also wish to thank the production and quality-control staff at Geneva's blood transfusion centre, and Thomas Lecompte for helpful discussions.

\section{References}

1 Franco AT, Corken A, Ware J. Platelets at the interface of thrombosis, inflammation, and cancer. Blood 2015;126(05):582-588

2 Meyer J, Lejmi E, Fontana P, Morel P, Gonelle-Gispert C, Bühler L. A focus on the role of platelets in liver regeneration: do plateletendothelial cell interactions initiate the regenerative process? J Hepatol 2015;63(05):1263-1271

3 Semple JW, Italiano JE Jr, Freedman J. Platelets and the immune continuum. Nat Rev Immunol 2011;11(04):264-274

4 Landry P, Plante I, Ouellet DL, Perron MP, Rousseau G, Provost P. Existence of a microRNA pathway in anucleate platelets. Nat Struct Mol Biol 2009;16(09):961-966

5 Nagalla S, Shaw C, Kong X, et al. Platelet microRNA-mRNA coexpression profiles correlate with platelet reactivity. Blood 2011;117(19):5189-5197

6 Zufferey A, Ibberson M, Reny JL, et al. New molecular insights into modulation of platelet reactivity in aspirin-treated patients using a network-based approach. Hum Genet 2016;135(04):403-414

7 Sunderland N, Skroblin P, Barwari T, et al. MicroRNA biomarkers and platelet reactivity: the clot thickens. Circ Res 2017;120(02):418-435

8 de Boer HC, van Solingen C, Prins J, et al. Aspirin treatment hampers the use of plasma microRNA-126 as a biomarker for the progression of vascular disease. Eur Heart J 2013;34(44):3451-3457

9 Leierseder S, Petzold T, Zhang L, Loyer X, Massberg S, Engelhardt S. MiR-223 is dispensable for platelet production and function in mice. Thromb Haemost 2013;110(06):1207-1214

10 Schober A, Nazari-Jahantigh M, Wei Y, et al. MicroRNA-126-5p promotes endothelial proliferation and limits atherosclerosis by suppressing Dlk1. Nat Med 2014;20(04):368-376

11 Kaudewitz D, Skroblin P, Bender LH, et al. Association of microRNAs and YRNAs with platelet function. Circ Res 2016;118(03): 420-432

12 Teruel-Montoya R, Kong X, Abraham S, et al. MicroRNA expression differences in human hematopoietic cell lineages enable regulated transgene expression. PLoS One 2014;9(07):e102259

13 Hong W, Kondkar AA, Nagalla S, et al. Transfection of human platelets with short interfering RNA. Clin Transl Sci 2011;4(03): 180-182

14 Blin A, Le Goff A, Magniez A, et al. Microfluidic model of the platelet-generating organ: beyond bone marrow biomimetics. Sci Rep 2016;6:21700

15 Strassel C, Brouard N, Mallo L, et al. Aryl hydrocarbon receptordependent enrichment of a megakaryocytic precursor with a high potential to produce proplatelets. Blood 2016;127(18):2231-2240

16 Schlinker AC, Duncan MT, DeLuca TA, Whitehead DC, Miller WM. Megakaryocyte polyploidization and proplatelet formation in low-attachment conditions. Biochem Eng J 2016;111:24-33
17 De Bruyn C, Delforge A, Martiat P, Bron D. Ex vivo expansion of megakaryocyte progenitor cells: cord blood versus mobilized peripheral blood. Stem Cells Dev 2005;14(04):415-424

18 Pineault N, Robert A, Cortin V, Boyer L. Ex vivo differentiation of cord blood stem cells into megakaryocytes and platelets. Methods Mol Biol 2013;946:205-224

19 Choi ES, Nichol JL, Hokom MM, Hornkohl AC, Hunt P. Platelets generated in vitro from proplatelet-displaying human megakaryocytes are functional. Blood 1995;85(02):402-413

20 Panuganti S, Schlinker AC, Lindholm PF, Papoutsakis ET, Miller WM. Three-stage ex vivo expansion of high-ploidy megakaryocytic cells: toward large-scale platelet production. Tissue Eng Part A 2013;19(7-8):998-1014

21 Kamat V, Muthard RW, Li R, Diamond SL. Microfluidic assessment of functional culture-derived platelets in human thrombi under flow. Exp Hematol 2015;43(10):891-900

22 Van Aelst B, Feys HB, Devloo R, Vandekerckhove P, Compernolle V. Microfluidic flow chambers using reconstituted blood to model hemostasis and platelet transfusion in vitro. J Vis Exp 2016;(109):

23 Zampetaki A, Willeit P, Tilling L, et al. Prospective study on circulating microRNAs and risk of myocardial infarction. J Am Coll Cardiol 2012;60(04):290-299

24 Kok MG, Halliani A, Moerland PD, Meijers JC, Creemers EE, PintoSietsma SJ. Normalization panels for the reliable quantification of circulating microRNAs by RT-qPCR. FASEB J 2015;29(09):3853-3862

25 Vandesompele J, De Preter K, Pattyn F, et al. Accurate normalization of real-time quantitative RT-PCR data by geometric averaging of multiple internal control genes. Genome Biol 2002;3(07): $\mathrm{H} 0034$

26 Otsu N. A threshold selection method from gray-level histograms. IEEE Trans Syst Man Cybern 1979;9(01):62-66

27 Meyer F. Topographic distance and watershed lines. Signal Processing 1994;38(01):113-125

28 Wang CZ, Yuan P, Li Y. MiR-126 regulated breast cancer cell invasion by targeting ADAM9. Int J Clin Exp Pathol 2015;8(06): 6547-6553

29 Jiang J, Woulfe DS, Papoutsakis ET. Shear enhances thrombopoiesis and formation of microparticles that induce megakaryocytic differentiation of stem cells. Blood 2014;124(13): 2094-2103

30 Bhatlekar S, Nagalla S, Lindsey C, et al., eds. MiR-125a-5p Regulates Megakaryocyte Differentiation and Proplatelet Formation. Berlin: International Society of Thrombosis and Haemostasis; 2017

31 Garzon R, Pichiorri F, Palumbo T, et al. MicroRNA fingerprints during human megakaryocytopoiesis. Proc Natl Acad Sci U S A 2006;103(13):5078-5083

32 Opalinska JB, Bersenev A, Zhang Z, et al. MicroRNA expression in maturing murine megakaryocytes. Blood 2010;116(23):e128-e138

33 Savage B, Saldívar E, Ruggeri ZM. Initiation of platelet adhesion by arrest onto fibrinogen or translocation on von Willebrand factor. Cell 1996;84(02):289-297

34 Maxwell MJ, Westein E, Nesbitt WS, Giuliano S, Dopheide SM, Jackson SP. Identification of a 2-stage platelet aggregation process mediating shear-dependent thrombus formation. Blood 2007; 109(02):566-576

35 Eichinger CD, Fogelson AL, Hlady V. Functional assay of antiplatelet drugs based on margination of platelets in flowing blood. Biointerphases 2016;11(02):029805

36 Xiang G, Cheng Y. MiR-126-3p inhibits ovarian cancer proliferation and invasion via targeting PLXNB2. Reprod Biol 2018;18(03): 218-224

37 Zhu L, Bergmeier W, Wu J, et al. Regulated surface expression and shedding support a dual role for semaphorin $4 \mathrm{D}$ in platelet responses to vascular injury. Proc Natl Acad Sci U S A 2007;104 (05):1621-1626

38 Wannemacher KM, Wang L, Zhu L, Brass LF. The role of semaphorins and their receptors in platelets: lessons learned from neuronal and immune synapses. Platelets 2011;22(06):461-465 
39 Wannemacher KM, Jiang H, Hess PR, Shin Y, Suzuki-Inoue K, Brass LF. An expanded role for semaphorin 4D in platelets includes contactdependent amplification of Clec- 2 signaling. J Thromb Haemost 2013;11(12):2190-2193

40 Le AP, Huang Y, Pingle SC, et al. Plexin-B2 promotes invasive growth of malignant glioma. Oncotarget 2015;6(09):7293-7304
41 Roney KE, O'Connor BP, Wen $\mathrm{H}$, et al. Plexin-B2 negatively regulates macrophage motility, Rac, and Cdc42 activation. PLoS One 2011;6(09):e24795

42 Agarwal V, Bell GW, Nam JW, Bartel DP. Predicting effective microRNA target sites in mammalian mRNAs. eLife 2015; $4: 4$ 